\title{
A Hybrid Approach for Autism Spectrum Disorder Classification
}

\author{
V. Jalaja Jayalakshmi ${ }^{1}$ and V. Geetha ${ }^{1}$ \\ ${ }^{1}$ Department of Computer Applications, Kumaraguru College of Technology, Coimbatore
}

\begin{abstract}
Autism spectrum disorder (ASD) is a neurological condition that can be devastating to the social functioning of the affected person. It is attributed to a range of symptoms that include troubles in social interaction, difficulty in expressing themselves and repetitive pattern filled behavior. People with autism have a unique behavioral pattern and the severity of the disease may vary across individuals, the causes for which are not known. The prevalence of ASD is increasing globally and early diagnosis of the disorder can lead to substantial behavioral improvements. Machine learning techniques are widely used in the health care domain for medical diagnosis. The study focuses on applying machine learning ensemble techniques to autism adult data sets to predict autism in adults. The UCI Machine Learning Repository's Autistic Spectrum Disorder Screening Data for Adult was used for the experiment purpose. The hybrid approach makes use of rough set algorithms for feature selection using Rosetta rough set tool and Adaboost with decision stump for classification using Weka data mining tool. Classification accuracy was high when the dataset was selected based on the reducts generated by Genetic algorithm. Results indicate that the proposed hybrid model improves the performance of autism data classification.
\end{abstract}

KEY WORDS: AUTISM, ENSEMBLE METHODS, MACHINE LEARNING, REDUCTS, ROUGH SET.

\section{INTRODUCTION}

Autism Spectrum Disorder (ASD) is a complex developmental disorder associated with symptoms that involve "persistent defects across multiple contexts in social communication and social interaction" and "limited, repetitive behavior, interest, or activity patterns". Autism is classified as a "spectrum" condition because the form and nature of symptoms that people encounter differ widely. Throughout all ethnic, racial, and economic groups, ASD occurs. While ASD can be a lifelong

\section{ARTICLE INFORMATION}

*Corresponding Author: jalajajayalakshmi.v.mca@kct.ac.in Received 9th Oct 2020 Accepted after revision 23rd Nov 2020 Print ISSN: 0974-6455 Online ISSN: 2321-4007 CODEN: BBRCBA

Thomson Reuters ISI Web of Science Clarivate Analytics USA and Crossref Indexed Journal

\section{Clarivate
Analytics}

NAAS Journal Score 2020 (4.31) SJIF: 2020 (7.728)

A Society of Science and Nature Publication,

Bhopal India 2020. All rights reserved.

Online Contents Available at: http//www.bbrc.in/

Doi: http://dx.doi.org/10.21786/bbrc/13.11/3 condition, the symptoms and capacity to function of an individual can be improved by therapies and services.

Diagnosis during the early stages of life significantly enhances the future of children with autism, by allowing for therapy when the brain of the child is still rapidly developing. People with autism have a unique behavioral pattern and the severity of the disease may vary across individuals, the causes for which are not known. The prevalence of ASD is increasing globally and early diagnosis of the disorder can lead to substantial behavioral improvements.

Machine learning is an application that gives systems the capability to learn and develop automatically from knowledge without being specifically programmed. Machine learning offers smart alternatives to the study of large data volumes. Machine Learning can generate precise results and analysis by designing quick and efficient algorithms and data-driven models for real-time data processing.

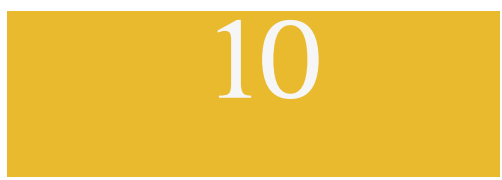


In enhancing the efficacy of behavioral health screeners, machine learning can play a vital role (Halim Abbas et al, 2018). In several observational studies on autism data sets (Tabtah F, 2017), machine learning approaches have been commonly used. Machine learning has great capabilities to improve the analytical and intervention research in the behavioral sciences and may be especially useful in investigations concerning the highly widespread and varied syndrome of autism spectrum disorder (Bone et al, 2015). Support Vector Machines, k-nearest neighbour \& Random Forest (RF) were applied on the autism data set and the results indicate that RF has got better performance in terms of accuracy measure. Weighted decision tree prediction model has been proposed for Autism Risk Analysis (Mythili et al., 2016).

Suman Raj et al (2020) strongly recommend implementing a CNN based model detection of Autism Spectrum Disorder. Accuracy of prediction of Autism Spectrum Disorder by Multilayer Perceptron classifier is better than the well-known algorithms as suggested by the authors (Jalaja et al., 2019). A study (Aboul Ella Hassanien et al., 2008) indicates that rough sets can be used for inductive learning and for constructing expert systems. Rough set theory has algorithms for knowledge reduction, concept approximation, decision rule induction, and object classification (Prerna Mahajan et al., 2012). The authors have applied a hybrid data mining model and ensemble learning classifiers to predict the credit scoring in banking domain (Koutanaei et al., 2015). Rough set-based ensemble algorithms has been used on four agricultural data sets and the results how that these algorithms perform better than the existing algorithms (Shi et al., 2018).This paper recommends a hybrid approach using rough set theory and Ada boosting algorithms for classifying the autism data set.

The organization of the paper is as follows: Section 2 describes about the algorithms used in the proposed methodology. The Experimental setup and results obtained are examined in Section 3. The concluding remarks and future work are given in Section 4.

\section{MATERIAL AND METHODS}

This work proposes a rough set-based ensemble model for effective classification of autism data set. The hybrid approach makes use of rough set algorithms for feature selection using Rosetta rough set tool and Adaboost with decision stump for classification using Weka data mining tool. The basic concepts of various algorithms used in this work are discussed below:

Rough Sets: Rough sets, proposed by Pawlak, is a mathematical tool for analyzing data. It has efficient procedures for finding hidden patterns of data. Rough set theory has algorithms for attribute selection and extraction, data reduction using reducts, rule generation, and pattern extraction. It stores data in tabular form, with each row representing an object. The table contains conditional attributes and a decision attribute and is known as an information system or a decision table.
If many objects in the decision table contain the same attribute values, then only one representative object is chosen, thus removing the redundant objects. These objects are known as indiscernible objects. Rough set makes use of lower and upper approximations for representation of a given set, if the available information is not adequate to decide the precise value of the set. This method of using approximation is the main idea of rough set theory. If all the objects surely belong to the set, it is known as lower approximation and if they possibly belong to the set, it is called as upper approximation.

Feature Selection: The desired features of the decision table in rough sets are selected using reducts. Reducts are the subset of conditional attributes which are adequate to categorize the decision table. The attributes that maintain the indiscernibility relation are only considered and there might be several such subset of attributes. Reducts contain the minimal attributes. The proposed work uses Johnson's and genetic algorithms for generating reducts.

Johnson's Algorithm: This algorithm is used for generating reducts using a heuristic approach. The algorithm takes the discernibility matrix as the input and counts the occurrence of each attribute. The attribute that occurs the highest number of times is added to the reduct candidate set which is initially empty. After adding the attribute to the reduct set, all the cells that contain the attribute are removed from the matrix. This process is iterated until all the non-empty cells are eliminated. This algorithm will return only a single reduct as shown in Figure 1.

\begin{tabular}{|c|c|c|}
\hline \multicolumn{2}{|c|}{ II Johnson Reduct } & \multirow{2}{*}{\begin{tabular}{|l|l|l|}
0 & 0 & $x$ \\
Support Lengtt
\end{tabular}} \\
\hline & Reduct & \\
\hline 1 & 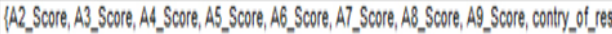 & 1009 \\
\hline
\end{tabular}

Figure 2: Reducts Generated By Genetic Algorithm

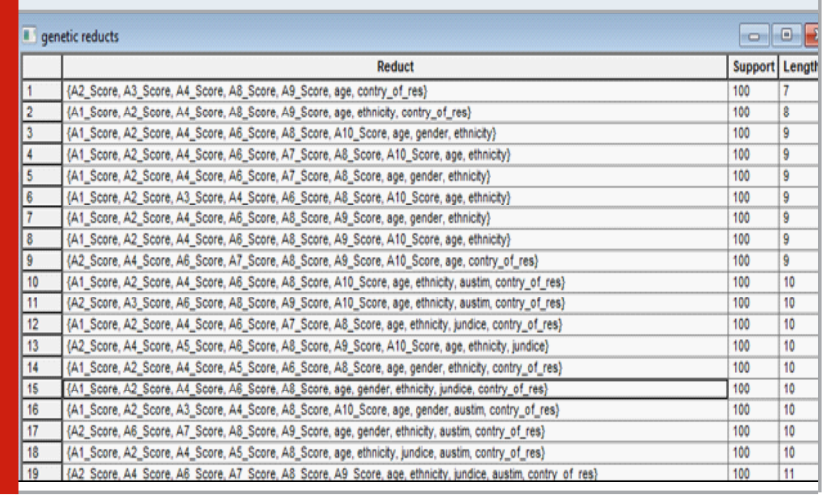

Genetic Algorithm: Genetic algorithms are methods of optimization based on the concepts of evolution. This is a stochastic approach focused on the mechanics of natural genetics and biological evolution for function optimization. To generate better approximations, genetic 
algorithms function on a population. A new population is produced at each generation by selecting individuals in the problem domain according to their level of fitness and recombining them together using operators borrowed from natural genetics. This algorithm is used to generate the best possible set of reducts for the Autism Adult data set and is shown in the Figure 2.

Adaboost: Ensemble methods is a powerful machine learning paradigm that merges predictions from several models to boost the performance of classification algorithms. Bagging and boosting are ensemble methods in machine learning algorithms. Decision trees are one of the widely used classification algorithms but tend to suffer from bias and variance Ada boost in an iterative ensemble method which combines several weak classifiers to build a single strong classifier. It initially builds a model from the training data. Then it creates a next model by correcting the errors in the first model. The process is repeated until the training set predicts correctly or the maximum model gets added. The various versions of the model use the technique of bootstrapping in which the samples are selected with replacement.

\section{RESULTS AND DISCUSSION}

Data Description: The UCI Machine Learning Repository's Autistic Spectrum Disorder Screening Data for Adult (Dua D and Graff C, 2019) was used for the experiment purpose. The dataset consists of 21 variables with 10 behavioural features, 10 individual characteristics and one class variable denoting if the person has Autism or not.

Data Pre-processing: The dataset consists of 704 instances. The data is preprocessed to enhance the quality of the data. The data cleaning step is used to remove noise and correct the inconsistencies in the data. In the dataset, the variables 'ethnicity' and 'relation' both had missing values in 95 records. 86 out of 95 are with class 'NO' and 9 out 95 are with class 'YES'. Since the variables with missing values are of categorical type, it is difficult to replace the missing value. Hence the 95 records were removed.

A record with outlier in the 'age' column was found with the value 383 , which is not possible. Considering it as a typo error, the age with 383 was replaced with 38 . After data cleaning, out of the 609 records, 180 records were with class value 'yes' and 429 records were with class value 'NO'. The variables 'age_desc' and 'used_app' were removed since these had the same value in all the records. After replacing the missing values and removing the unwanted attributes, rough set concepts from the Rosetta rough set tool was used for discretization and feature selection. The process of translating the values of continuous data attributes into a limited set of intervals and associating certain data values with each interval is data discretization.

Rosetta rough set tool has various algorithms for discretization and equal frequency binning algorithm was chosen to be applied on the autism data set. Equal frequency binning algorithm splits the data into ' $k$ ' number of bins and all the bins will have equal number of values. Once discretization is complete, feature selection methods are applied on the data set. These methods are used to increase the performance of classifier algorithms. In rough set theory feature selection is carried out by the generation of reducts using rough set algorithms. The entire framework for data preprocessing is shown in Figure 3.

Ensemble methods on Autism data set: Ensemble methods have been used on autism adult data set for finding decision rules to predict the occurrence of Autism Spectrum Disorder using Weka data mining tool. The attributes from the data set were selected based on the reducts generated by the rough set algorithms. The updated data set was randomly split into training and test data using varying split factors. Then Ada boosting algorithms with decision stump were used on the training data set. The rules generated were then validated with the test data set. The number of rules generated by genetic algorithm is much larger than that generated by Johnson's algorithm. The steps in the application of ensemble methods on the decision tree is shown in Figure 4.

Figure 3: Framework For Data Preprocessing

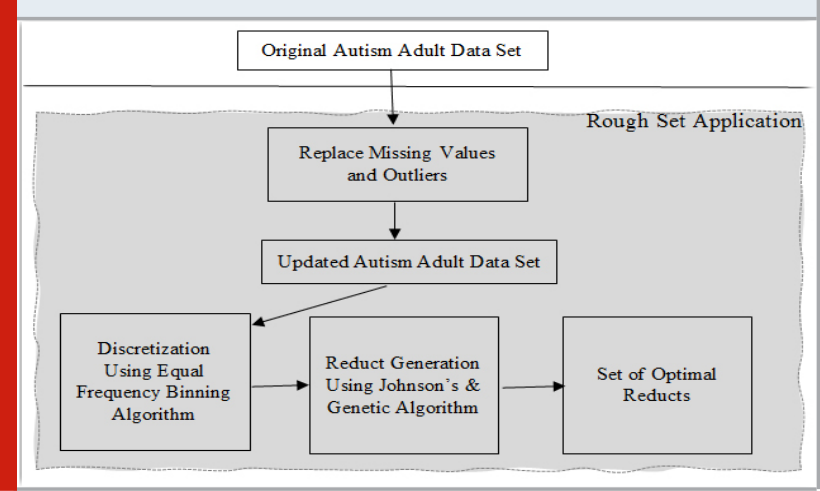

Figure 4: Framework Of The Proposed Approach

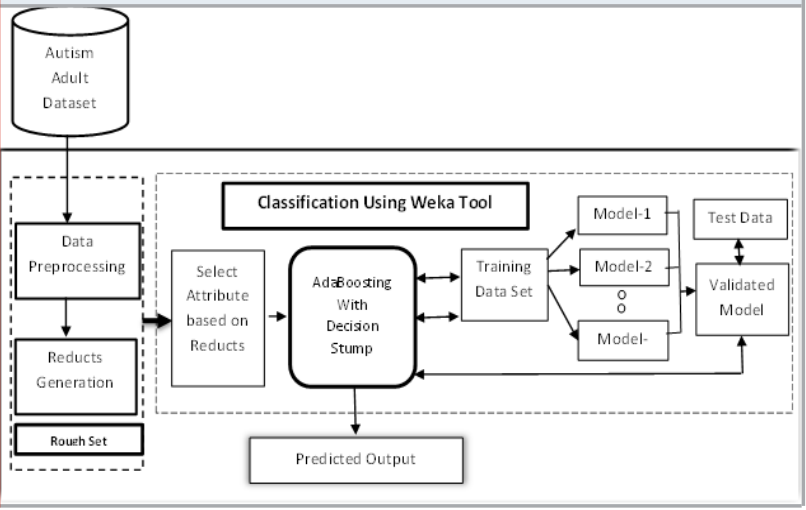

Evaluation of the Proposed Model: The performance of the model was evaluated in terms of accuracy, Kappa Statistics and F-measure. The results obtained using Ada boosting algorithms using the attributed selected from 
reducts generated by Johnson's algorithm and Genetic algorithms were compared with the application of the same algorithm on the data set without using reducts and the results are discussed below.

Accuracy: Accuracy refers to the correctly classified instances for the given test data set and the results are shown in Figure 5.The results indicate when the training samples are less ( $10 \%$ \&t 20\% ), the performance of Adaboost algorithm without feature selection is better but when the number of training samples increase ( 30 $\%$ to $80 \%$ ), the reduction of attributes has improved the prediction accuracy. When compared to Johnson's algorithm, the genetic algorithm performs better. This is because genetic algorithms generate a greater number of reducts than Johnson's algorithm.

Figure 5: Accuracy Percentage Of Different Classifiers

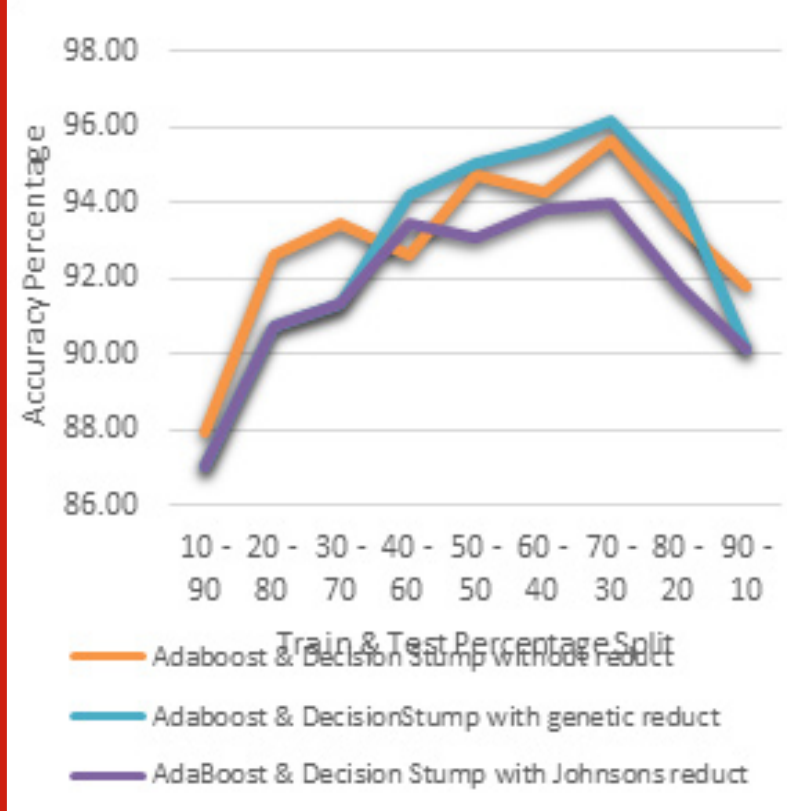

Table 1. Kappa Statistics Of Classifiers

\begin{tabular}{|l|c|c|}
\hline $\begin{array}{l}\text { Training-test } \\
\text { data split } \\
\text { percentage }\end{array}$ & $\begin{array}{c}\text { Adaboost } \mathrm{Ct} \\
\text { Decision Stump } \\
\text { without reduct }\end{array}$ & $\begin{array}{c}\text { Adaboost } \mathrm{Ct} \\
\text { Decision Stump } \\
\text { with genetic reduct }\end{array}$ \\
\hline $10-90$ & 0.702 & 0.696 \\
\hline $20-80$ & 0.829 & 0.778 \\
\hline $30-70$ & 0.846 & 0.795 \\
\hline $40-60$ & 0.828 & 0.864 \\
\hline $50-50$ & 0.880 & 0.886 \\
\hline $60-40$ & 0.863 & 0.889 \\
\hline $70-30$ & 0.889 & 0.902 \\
\hline $80-20$ & 0.847 & 0.867 \\
\hline $90-10$ & 0.822 & 0.788 \\
\hline
\end{tabular}

Figure 6: Comparison of F-Measure Values Between The Two Classifiers

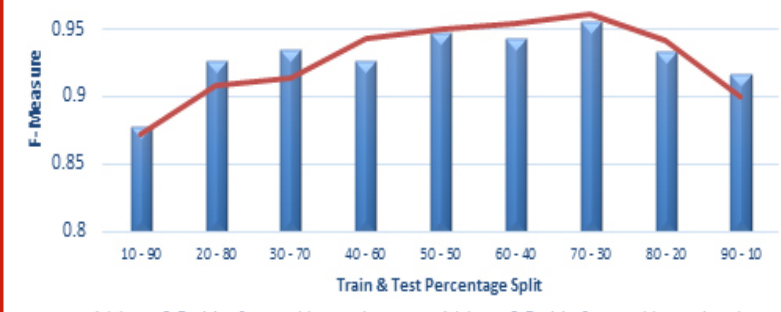

Adaboost \& Decision Stump without reduct — Adaboost \& DecisionStump with genetic reduct

Kappa Statistics: Kappa Statistic is a measure of the agreement between the predicted and the actual classifications in a dataset and the values lie between -1 to 1 . A Kappa statistic value greater than 0.7 is usually considered as a good statistic correlation, but higher the value, the better the correlation. Table 1 helps to visualize the interpretation of Kappa value. It can be clearly inferred from Table.1. that using reducts has the highest Kappa statistic 0.90 and the lowest 0.696, which indicates a high to moderate degree of acceptance. The Kappa value ranges from 0.702 to 0.889 when reducts are not used. The agreement of prediction given by Kappa statistic is highest when Adaboost algorithm is applied on the reduced data set, while it is the lowest in dataset without reducts.

F-Measure: F-measure is a weighted combination of precision and recall. It finds the proportion of the true positives to the mean of predicted positives and real positives. The F-measure blends precision and recall into a single performance measure. The F-measure value varies from 0 to 100\% and a higher F-measure value implies a better quality of the classification. A system with high F-measure has good precision and recall. The results based on F-measure with and without using reducts are compared and is shown in Figure 6. The quality of the best F-measure result of Adaboost with reduct (0.961\%) is achieved for 70\% training samples. This is higher than the algorithm without using reducts by $0.5 \%$. This indicates that there is not much significant difference of F-measure values between both the algorithms.

\section{CONCLUSION \& FUTURE WORK}

A new hybrid model has been designed for Autism detection using Rough set feature selection and ensemble learning classifiers. Two feature selection algorithms of rough set theory were applied to obtain a set of suitable subset of features, which gives a good classification performance when Adaboost learning classifier was used. Among the feature selection algorithms, genetic algorithm has given a better performance. Classification accuracy was high when the dataset was selected based on the reducts generated by Genetic algorithm. The proposed hybrid model can be used to predict autism spectrum disorder at an early stage to provide an immediate treatment. As a part of future work, other 
feature selection algorithms can be applied on the data set and tested.

\section{REFERENCES}

Abbas H Garberson F Glover E and Wall DP (2018) Machine Learning Approach for Early Detection of Autism by Combining questionnaire and home video screening, Journal of the American Medical Informatics Association, 25(8) pp.1000-1007.

Bone D et al., (2015) Applying Machine Learning to Facilitate Autism Diagnostics: Pitfalls and Promises, J Autism Dev Disorf 45(5), pp.1121-1136.

Dua D and Graff C (2019) UCI Machine Learning Repository [http://archive.ics.uci.edu/ml]. Irvine, CA: University of California, School of Information and Computer Science.

Hassanien AE Abdelhafez ME and Own HS (2008) Rough Sets Data Analysis in Knowledge Discovery: A Case of Kuwaiti Diabetic Children Patients, Advances in Fuzzy Systems, Volume 2008, pp.1-13.

Jalaja Jayalakshmi V Geetha V and Vivek R (2019) Classification of Autism Spectrum Disorder Data using Machine Learning Techniques, International Journal of Engineering and Advanced Technology (IJEAT), ISSN: 2249 - 8958, Volume-8 Issue-6S, pp.565-569.
Koutanaei FN Sajedi H and Khanbabaei M (2015) A Hybrid Data Mining Model of Feature Selection Algorithms and Ensemble Learning Classifiers for Credit Scoring, Journal of Retailing and Consumer Services, vol. 27, pp.11-23.

Mahajan P, Kandwal R and Vijay R (2012) Rough Set Approach in Machine Learning: A Review, International Journal of Computer Applications 56(10), pp.1-13.

Mythili MS and Mohamed Shanavas AR (2016) An Improved Autism Predictive Mechanism among Children Using Fuzzy Cognitive Map and Feature Extraction Methods (Feast), ARPN Journal Of Engineering And Applied Sciences, Vol. 11, No. 3, pp.1451-1456.

Raj S and Masood S (2020) Analysis and Detection of Autism Spectrum Disorder Using Machine Learning Techniques”, Procedia Computer Science ,167, pp.9941004.

Shi L Duan Q Zhang J Xi L and Ma X (2018) Rough Set based Ensemble Learning Algorithm for Agricultural Data Classification, Filomat 32(5), pp.1917-1930.

Tabtah F (2017) Autism Spectrum Disorder Screening: Machine Learning Adaptation and DSM-5 Fulfillment", Proceedings of the 1st International Conference on Medical and Health Informatics 2017, Taichung City, Taiwan, ACM., pp.1-6. 